\title{
Introduction du tome 1
}

\subsection{But de l'ouvrage}

Ce livre présente une introduction aux principaux concepts et outils communs à la physique statistique et à la théorie quantique des champs : développements perturbatifs et diagrammes de Feynman, intégrales de chemin et intégrales fonctionnelles, théorie de la renormalisation et groupe de renormalisation.

Ces concepts et ces techniques mathématiques sont apparus à partir des années 1940-1950 à la fois en physique des hautes énergies (QED, théorie de la renormalisation, théories de jauge non abéliennes), en physique du problème à $\mathrm{N}$-corps (physique nucléaire, physique de la matière condensée) et en physique statistique. Ces développement croisés ont culminé au début des années 1970 avec les applications du groupe de renormalisation à la fois (1) en physique des hautes énergies : construction du modèle standard des interactions électrofaibles et de la chromodynamique quantique, liberté asymptotique, et (2) en physique statistique par la théorie moderne des phénomènes critiques : les transitions de phase continues et les comportements critiques associés sont en fait décrits par des théories quantiques des champs!

Depuis ces idées et ces méthodes théoriques se sont appliquées à de très nombreux domaines de la physique statistique (phénomènes critiques, systèmes désordonnés, phénomènes hors équilibre, processus de croissance), de la physique de la matière condensée (physique des solides, matière molle, systèmes mésoscopiques), de la physique des systèmes quantiques (atomes froids), des systèmes dynamiques (transition vers le chaos, turbulence, systèmes complexes), pour citer les principaux. Elles sont en train d'irriguer et d'inspirer des domaines importants des mathématiques. Elles sont regroupées souvent sous le terme de « théorie statistique des champs ». Plutôt qu'une théorie comme la relativité ou la mécanique quantique, la théorie statistique des champs est une «boîte à outils » (outils venus de la physique statistique et de la physique quantique) dont le contenu est maintenant indispensable au physicien théoricien.

Ces succès reposent sur deux éléments.

(1) Tout d'abord il existe une analogie profonde entre le traitement mathématique des fluctuations thermiques en physique statistique et celui des «fluctuations quantiques » (principe d'incertitude) en physique quantique. Cette 
analogie est particulièrement claire dans la formulation de la mécanique quantique en termes d'intégrale de chemins (Feynman). Une intégrale de chemin en « temps imaginaire » est analogue à une somme sur les micros états d'un système statistique classique $1 \mathrm{D}$ dans l'ensemble grand canonique, la constante de Planck $\hbar$ jouant le rôle de la température $T$. Cette analogie se généralise très naturellement entre les champs quantiques en $D$ dimensions d'espace et les systèmes statistiques étendus en $D+1$ dimensions.

(2) Ensuite, les théories quantiques des champs (en général) et les phénomènes critiques sont des systèmes physiques avec un très grand nombre de degrés de liberté indépendants où les fluctuations (quantiques et statistiques) sont importantes sur une très grande gamme d'échelles de distance (longeur d'onde) et de fréquence (énergie). Leurs couplages et leur influence sur la dynamique « effective »du système ne peuvent être traités simplement. La théorie du groupe de renormalisation permet précisément de contrôler - plus ou moins proprement - ces couplages multi-échelles, en définissant proprement le concept de « théorie effective » et en permettant de calculer les « couplages effectifs ». Elle permet de dégager quels sont les degrés de liberté importants (en théorie quantique quels sont les champs) pour décrire la dynamique d'un système à une échelle donnée. De ce point de vue, le groupe de renormalisation a révolutionné notre façon d'aborder de nombreux problèmes en physique (nature des interactions fondamentales, émergence de comportements complexes, apparition de lois d'échelles) et au-delà.

Il faut aussi mentionner d'autres idées très importantes qui font partie de cette boîte à outil, en particulier dans l'étude des systèmes de basse dimensionnalité et des systèmes désordonnés : excitations topologiques (solitons, vortex, instantons) et effets non perturbatifs, solutions exactes et systèmes intégrables, invariance conforme, supersymétrie... Elles forment le socle de la théorie des cordes. Elles sont également à l'origine des contacts et de la fertilisation croisée entre la théorie quantique des champs et les mathématiques.

Enfin un certain nombre de méthodes de discrétisation (théories sur réseau, développements de couplage fort) et de méthodes de simulations numériques (Monte-Carlo), venues de la physique statistique, sont devenues des outils standards en théorie quantique des champs et en physique des hautes énergies.

\subsection{Contenu de l'ouvrage}

Cet ouvrage est donc une introduction aux applications de la théorie des champs à la mécanique statistique. Son contenu est cohérent, il peut être utilisé seul, ainsi que comme une introduction à certains aspects de la physique des champs et des particules, et à la physique statistique des systèmes à l'équilibre. Il ne dispense pas de la pratique de traités de théorie quantique des champs pour la physique des hautes énergies, ni d'ouvrages consacrés à la physique statistique et à la physique de la matière condensée. 
Ce manuel est divisé en quatre grandes sections, et pour des raisons pratiques en deux tomes. Ce premier tome se compose des parties I et II, et traite plutôt des aspects théorie quantique et théorie quantique des champs. Le deuxième tome se composera des parties III et IV, et traitera tout d'abord des aspects 《statistique » et des applications en physique statistique de la théorie.

La partie I traite de l'intégrale de chemin en mécanique quantique. Le but de cette partie est de bien faire comprendre l'analogie entre physique statistique à l'équilibre et mécanique quantique (formalisme du temps imaginaire). Les chapitres 1, 2 et 3 en forment la partie essentielle. Les chapitres 4 et 5 présentent des aspects plus avancés. Ils peuvent être sautés en première lecture, et leurs différentes sections peuvent être lues indépendamment en général.

La partie II est une introduction à l'intégrale fonctionnelle en théorie quantique des champs, partir de l'exemple de la théorie scalaire $\phi^{4}$. Les formulations de la théorie à temps réel et à temps euclidien et les règles de Feynman pour construire la théorie des perturbations sont traitées dans les chapitres 6 et 7 . La théorie de la renormalisation perturbative et les équations du groupe de renormalisation sont introduites en détail au premier ordre dans le chapitre 8. Une brève introduction aux aspects plus généraux de la renormalisation perturbative est donnée dans le chapitre 9. Les formulations non perturbatives de la renormalisation «à la Wilson » et l'équivalence entre la théorie des champs $\phi^{4}$ renormalisée et la limite d'échelle au point critique du modèle d'Ising sont abordées dans le tome 2 .

La partie III traitera de la physique statistique des phénomènes critiques et de la théorie du groupe de renormalisation dans l'espace réel. Après un rappel des concepts de base de physique statistique, la théorie du champ moyen et la théorie de Laudau des phénomènes critiques seront introduites. Le principe de la théorie de K. Wilson du groupe de renormalisation et ses conséquences pour les phénomènes critiques seront ensuite explicités. Un chapitre sera consacré à des calculs explicites sur la théorie de Landau-Ginsburg-Wilson (LGW), essentiellement dans l'approximation du potentiel local. Ceci illustrera la puissance de la théorie de Wilson, et permettra de discuter en profondeur les relations entre renormalisation de Wilson et renormalisation perturbative en théorie des champs.

La partie IV présentera des applications physiques de la théorie statistique des champs en physique statistique et en physique de la matière condensée. Elle contiendra également une introduction à des aspects plus avancés et modernes : invariance conforme, effets de taille finie. Les différents exemples pourront s'étudier indépendamment.

En principe, les parties I (intégrale de chemin en mécanique quantique) et III (mécanique statistique, phénomènes critiques et groupe de renormalisation) peuvent être lues indépendamment (bien que pour la fin de la partie III il vaille mieux avoir vu I et le début de II). La partie II (théorie quantique des 
champs et renormalisation) peut être lue à la suite de I, et indépendamment de III.

Mettons en garde le lecteur à propos de ce qu'il ne trouvera pas, ou peu, dans cet ouvrage :

- une exposition détaillée aux méthodes numériques et de calcul formel (analyse des développements en séries de hautes tempéatures, simulations par Monte-Carlo, matrice de transfert);

- une introduction aux méthodes exactes (systèmes intégrables, ansatz de Bethe, matrice S);

- un traité sur l'invariance conforme et les techniques reliées (gaz de Coulomb, dualité) ;

— une introduction à la physique des systèmes désordonnés (un sujet en soi) ;

- un traité sur les approches mathématiques rigoureuses (théorie constructive des champs, groupe de renormalisation exact, théorie de la renormalisation à tous les ordres) ;

- une introduction au groupe de renormalisation pour les systèmes quantiques.

\subsection{Remerciements}

Ce livre est issu principalement des notes destinées aux étudiants du cours de deuxième année du parcours « Physique théorique »du Master « Concepts fondamentaux de la physique », que j'ai donné à l'École normale supérieure (et dans les locaux de l'université Denis Diderot) de 2001 à 2015. Je tiens à remercier tout particulièrement Jesper Jacobsen, qui m'a assisté pour construire et assurer les séances d'exercices, et m'a remplacé pour les cours à l'occasion pendant toutes ses années. Je suis également très reconnaissant à Édouard Brézin, à qui je dois l'opportunité d'avoir donné ce cours, ainsi qu'à Costas Bachas et Adel Bilal, qui ont coordonné ce parcours, et mes collègues enseignants et administratifs (en particulier Nicole Ribet et Mascia Reato). Cet ouvrage a également beaucoup bénéficié des cours que j'ai donnés dans d'autres établissements, à commencer par celui de théorie quantique des champs pour le programme Perimeter Scholars International du Perimeter Institute, que je donne depuis 2009, et de celui pour le programme doctoral de la Suisse romande que j'ai donné à l'Ecole polytechnique fédérale de Lausanne. Je tiens donc à remercier chaleureusement Neil Turok, Tibra Ali, David Gerson et Dan Wohn du Perimeter, ainsi que Frédéric Mila et Aurelio Bay de l'EPFL. Avoir pu enseigner dans de telles institutions est pour moi une chance inestimable.

Michèle Leduc et Michel Le Bellac m'ont encouragé à faire de ces notes un ouvrage. Je les remercie infiniment pour leur persévérance et leur patience... Merci à Michel pour le temps passé à relire mes notes. De nombreux collègues 
m'ont, à divers stades, encouragé, conseillé ou inspiré pour la préparation de mes cours et l'écriture de ces notes. Je ne saurais me les remémorer tous, mais je tiens à mentionner, outre Jesper, Costas et Adel déjà cités, Denis Bernard, Michel Bauer, Olivier Parcollet et Jean Zinn-Justin.

Enfin, mes remerciements et ma gratitude vont bien sûr à tous les étudiants et les étudiantes qui ont suivi mes cours, pour leur intérêt, leur patience, leurs questions et leurs critiques. Ce sont eux qui ont été ma principale source de motivation et d'inspiration pour travailler sur ce cours et préparer ces notes.

\subsection{Bibliographie sommaire}

La littérature sur le sujet est très riche, et il existe déjà d'excellents ouvrages d'introduction à la physique quantique, la physique statistique, la théorie des champs et la théorie statistique des champs. Cet ouvrage essaye d'avoir une présentation quelque peu originale du sujet, surtout en ce qui concerne les relations entre groupe de renormalisation perturbatif et groupe de renormalisation wilsonien, mais je me suis inspiré consciemment ou inconsciemment de plusieurs ouvrages et cours que j'ai eu l'occasion de suivre.

\section{Ouvrages en français}

En mécanique classique, les livres de L.D. Laudau et E.M. Lifchitz [LL94] et le livre de V. Arnold [Arn74] sont des ouvrages de base. Pour la mécanique quantique, les deux volumes incontournables de C. Cohen-Tannodji, B. Diu et F. Laloë [CTDL73a] et le récent troisième volume de C. Cohen-Tannoudji, F. Laloë et B. Diu [CTLD17], sont des références ainsi que le livre de M. Le Bellac [LB13a, LB13b]. Pour une introduction à la mécanique statistique, on peut citer les deux volumes de cours de R. Balian [Bal82, Bal94]. Le livre de J. Zinn-Justin [ZJ12] est une introduction détaillée à l'intégrale de chemin en mécanique quantique.

Le classique (un peu ancien) ouvrage de C. Itzykson et J.-B. Zuber [ID13] est une bonne introduction à la théorie quantique des champs, plutôt du point de vue physique des hautes énergies.

Les deux volumes (un peu plus récents) de C. Itzykson et J.-M.Drouffe [ID13, ID89], et de M. Le Bellac [LB12] sont des introductions classiques à la théorie statistique des champs.

\section{Ouvrages en anglais}

Si on considère l'anglais, qui est la lingua franca de la communauté scientifique, et tend à le devenir pour l'enseignement pré-doctoral et doctoral, la littérature devient immense.

Les ouvrages en français précités sont pour la plupart disponibles en version anglaise : pour la mécanique quantique, le traité de Cohen-Diu-Lalo 
[CTDL92], celui de M. Le Bellac [LB06], celui de J. Zinn-Justin [ZJ10] ; pour la théorie des champs, ceux de C. Itzykson et J.-B. Zuber [IZ12], de C. Itzykson et J.-M. Drouffe [ID91], et celui de M. Le Bellac [LBB91].

Des ouvrages très classiques sont pour la théorie quantique des champs (donc surtout orientés physique des hautes énergies) : le traité de S. Weinberg [Wei95] (le premier des trois volumes pour ce qui est traité ici), le livre de M.E. Peskin et D.V. Schroder [PS18]. Le livre J. Zinn-Justin [ZJ02] est une bible orientée à la fois vers la physique des hautes énergies et la physique statistique. Beaucoup moins rigoureux et moins complet mais stimulant est le livre de A. Zee [Zee10]. Et pour ne pas oublier l'école russe, citons l'inspirant livre de A. Polyakov [Pol87].

Pour la théorie statistique des champs et ses applications à la mécanique statistique, citons (outre le Zinn-Justin) le livre de G. Parisi [Par98] et plus courts mais plus récents, les ouvrages de E. Brézin [Bré10] et de J. Cardy [Car96]. Des ouvrages récents orientés vers la physique de la matière condensées sont ceux de A.M. Tsvelik [Tsv07], de E. Fradkin [Fra13] et la très complète introduction à la physique de la matière condensée de P.M. Chaitkin et T.C. Lubensky [CL00].

Des références de base sur les méthodes mathématiques pour la physique théorique, indispensables pour un lecteur peu familier avec les outils mathématiques utilisés dans cet ouvrage, sont les ouvrages classiques de L. Schwartz et D. Huet [SH82] et de R. Courant et D. Hilbert [CH08] (ancien mais actualisé), et le traité plus récent et plus moderne de $\mathrm{M}$. Stone et $\mathrm{P}$. Goldbart [SG09].

Des références plus précises ou plus avancées seront données à la fin des différents chapitres. 


\subsection{Plan structuré du tome 1}

Basique (première lecture)

1 Rappels de mécanique classique et quantique

2 Intégrale de chemin : introduction

3 Intégrale de chemin et physique statistique

6 Intégrale fonctionnelle : le champ

libre

$7 \phi^{4}$ : théorie des perturbations

$8 \phi^{4}$ : renormalisation à 1 boucle
Avancé (seconde lecture)

4 Intégrale de chemin : présentation générale

5 Bosons, fermions et spins

9 Renormalisation : théorie générale 
